\title{
Macaque Retina Contains an S-Cone OFF Midget Pathway
}

\author{
Karl Klug, ${ }^{1}$ Steve Herr, ${ }^{2}$ Ivy Tran Ngo, ${ }^{2}$ Peter Sterling, ${ }^{3}$ and Stan Schein ${ }^{1,2}$ \\ ${ }^{1}$ Brain Research Institute and ${ }^{2}$ Department of Psychology, University of California, Los Angeles, Los Angeles, California 90095-1563, and ${ }^{3}$ Department of \\ Neuroscience, University of Pennsylvania, Philadelphia, Pennsylvania 19104
}

\begin{abstract}
Psychophysical results suggest that the primate visual system is equally sensitive to both the onset and offset of short-wavelength light and that these responses are carried by separate pathways. However, physiological studies of cells in the retina and lateral geniculate nucleus find far fewer OFF-center than ON-center cells whose receptive-field centers are driven by short-wavelength-sensitive (S) cones. To determine whether S cones contact ON and OFF midget bipolar cells as well as (ON) "blue-cone bipolar" cells (Mariani, 1984), we examined 118 contiguous cone terminals and their bipolar cells in electron micrographs of serial sections from macaque foveal retina. Five widely spaced cone terminals do not contact $\mathrm{ON}$ midget bipolar cells. These five cone terminals contact the dendrites of "blue-cone bipolar" cells instead, showing that they are the terminals of S cones. These S-cone terminals are smaller and contain more synaptic ribbons than other terminals. Like neighboring cones, each $S$ cone contacts its own OFF midget bipolar cell via triad-associated (flat) synaptic contacts. Moreover, each S-cone OFF midget bipolar cell has a synaptic terminal in the outer half of the inner plexiform layer, where it contacts an OFF midget ganglion cell.
\end{abstract}

Key words: cone; S cone; blue cone; retina; bipolar; retinal ganglion cell; midget; synapse; ribbon; color perception; retinal anatomy; retinal histology

\section{Introduction}

Psychophysical results suggest that the primate visual system is equally sensitive to both the onset and offset of short-wavelength light and that these responses are carried by separate pathways (Smith et al., 1989; DeMarco et al., 1994; Chichilnisky and Wandell, 1996; McLellan and Eskew, 2000). However, physiological studies of cells in the retina and lateral geniculate nucleus (LGN) find far fewer OFF-center cells than ON-center cells whose receptive-fields are driven by short-wavelength-sensitive (S) cones (Krüger, 1977; Malpeli and Schiller, 1978; de Monasterio, 1979; Zrenner and Gouras, 1981; Zrenner, 1983) (but see Valberg et al., 1986).

The scarcity of cells with S-cone OFF-center receptive fields was surprising because Golgi studies suggested that each foveal cone is presynaptic to one invaginating $(\mathrm{ON})$ and one flat (OFF) midget bipolar cell and that each midget bipolar cell is presynaptic to a single midget ganglion cell (Polyak, 1941; Boycott and Dowling, 1969; Kolb et al., 1969; Kolb, 1970). Moreover, physiologists had found approximately equal numbers of $\mathrm{ON}$ and $\mathrm{OFF}$ responses among ganglion cells and LGN cells with receptivefield centers dominated by middle- or long-wavelength-sensitive

Received May 8, 2003; revised Sept. 4, 2003; accepted Sept. 5, 2003.

This work was supported by National Institute of Mental Health Grant MH15795-18 and National Institutes of Health Grants EY11153 and EY08124. We thank Pooneh Esfahani for counting ribbons and for reconstructing cone terminals, Jack Ribble, Garrett Eggers, and Vanessa Preciado for help with bipolar and ganglion cell reconstructions, Dr. Yoshihiko Tsukamoto, Patricia Masarachia, and Sally Shrom for preparing the EM material, and Kazuki Uema and Lisa Travis for printing.

Correspondence should be addressed to Stan Schein, Department of Psychology, Franz Hall, Mail code 951563, University of California, Los Angeles, Los Angeles, CA 90095-1563. E-mail: schein@ucla.edu.

Copyright $\odot 2003$ Society for Neuroscience $\quad$ 0270-6474/03/239881-07\$15.00/0
(M or L) cones (Wiesel and Hubel, 1966; de Monasterio and Gouras, 1975). The rarity of cells with S-cone OFF-center receptive fields raised the possibility that $\mathrm{S}$ cones, unlike $\mathrm{L}$ and $\mathrm{M}$ cones, might not drive OFF midget bipolar and ganglion cells and that an S-cone-specific OFF circuit might not exist.

The picture of S-cone circuitry was further complicated by the discovery of a special "blue-cone bipolar" cell contacted exclusively by several S cones (Mariani, 1984; Kouyama and Marshak, 1992, 1997). This S-cone-specific ON bipolar cell provides the ON signals to a ganglion cell that has been described anatomically as a small bistratified cell and physiologically as receiving $\mathrm{ON}$ signals from $\mathrm{S}$ cones and coextensive OFF signals from $\mathrm{L}$ and $\mathrm{M}$ cones (Rodieck, 1991; Dacey, 1993; Dacey and Lee, 1994; Calkins et al., 1998; Chichilnisky and Baylor, 1999). The discovery of this $\mathrm{S}$-cone-specific $\mathrm{ON}$ bipolar cell raised another question: are $\mathrm{S}$ cones also presynaptic to ON midget bipolar cells?

We identified each cone and each midget and diffuse bipolar cell in a small region of macaque monkey foveal retina by reconstructing cells from serial sections (see Fig. $1 A$ ). S cones contact the distinctive $(\mathrm{ON})$ blue-cone bipolar cell described above (see Fig. $1 B$ ). Each L or M cone is presynaptic to its own ON midget bipolar cell, but none of the $\mathrm{S}$ cones are. In contrast, every cone, including every $\mathrm{S}$ cone, is presynaptic to an OFF midget bipolar cell. Like other midget bipolar cells, these S-cone OFF midget bipolar cells are presynaptic to midget ganglion cells. We conclude that $\mathrm{S}$ cones, like $\mathrm{L}$ and $\mathrm{M}$ cones, provide input to both $\mathrm{ON}$ and OFF cone-specific retinal circuits. The S-cone ON circuit is unique, with its own ON bipolar and ganglion cells, whereas the S-cone OFF circuit uses midget bipolar and ganglion cells.

Parts of this work have been published previously in abstract form (Klug et al., 1992, 1993; Esfahani et al., 1993). 


\section{Materials and Methods}

As described by Tsukamoto et al. (1992), a $6 \mathrm{~kg}$ adult male monkey (Macaca fascicularis) was given a preanesthetic dose of ketamine $(10 \mathrm{mg} /$ $\mathrm{kg}$ ) and a deep anesthetic dose $(40 \mathrm{mg} / \mathrm{kg})$ of pentobarbital, followed by perfusion through the aorta. Perfusion consisted of a brief $(20 \mathrm{sec})$ flush with saline, followed by $10 \mathrm{~min}$ of a solution containing $100 \mathrm{~mm} \mathrm{NaCl}, 30 \mathrm{~mm}$ sodium phosphate buffer, $\mathrm{pH} 7.40,2 \%$ glutaraldehyde, and $2 \%$ paraformaldehyde. The retina was removed and postfixed in $2 \% \mathrm{OsO}_{4}$ in $8 \%$ dextrose for $30 \mathrm{~min}$ at $4^{\circ} \mathrm{C}$ and $60 \mathrm{~min}$ at $22^{\circ} \mathrm{C}$. A piece of the retina containing the fovea was dissected, stained en bloc in $1 \%$ aqueous uranyl acetate, dehydrated, and embedded in Epon. A series of 319 consecutive ultrathin $(90 \mathrm{~nm})$ vertical sections were cut parallel to the horizontal meridian, through the fovea. The sections were mounted and then stained with uranyl acetate and lead citrate.

The region from 480 to $640 \mu \mathrm{m}$ nasal to the center of the retina was photographed en montage at 400 and $2000 \times$ magnification using an electron microscope and printed with $2.5 \times$ enlargement for final magnifications of 1000 and $5000 \times$. The outer plexiform layer and parts of the inner plexiform layer (IPL) were rephotographed at $5000 \times$ and enlarged $3.5 \times$ for $17,500 \times$ prints. Selected photoreceptor terminals were rephotographed at $10,000 \times$ and enlarged $2.5 \times$. Cells in this region connect to foveal cone inner segments centered around $1^{\circ}$ of visual eccentricity because of the lateral displacement of cone terminals from cone inner and outer segments (Schein, 1988; Tsukamoto et al., 1992). The region studied is well within foveal retina because the fovea extends to 500 $\mu \mathrm{m}$, or $2.5^{\circ}$ (Polyak, 1941).

Cone terminals, bipolar cells, and ganglion cells were tracked and reconstructed from the series of electron micrographs (see Fig. 2A). Each bipolar cell was classified as an ON or OFF midget bipolar, ON or OFF diffuse bipolar, rod bipolar, or blue-cone bipolar cell. Because the orientation of the sections is not perfectly vertical, midget bipolar and ganglion cells of some cones fall outside of the series and could not be completely reconstructed. For example, the midget bipolar cells contacted by cone terminals in the low-numbered sections at the bottom of Figure $1 \mathrm{~A}$ synapse with ganglion cells that are located in the high-numbered sections at the top of the same figure. Contours were traced from 5000 or $17,500 \times$ prints onto clear plastic sheets and digitized using the Montage software package (Smith, 1987). Some wire-frame models were tiled with the ContourFitter program (Meyers et al., 1992), visualized with Geomview (http://www.geomview.org/), and rendered with the Blue Moon Rendering Tools (NVIDIA, Santa Clara, CA). Montage was also used to calculate the volumes of 43 reconstructed cone terminals by summing volumes (surface area $\times$ section thickness) section by section.

\section{Results}

\section{$S$ cones do not contact $\mathrm{ON}$ midget bipolar cells}

We identified all of the midget bipolar cells postsynaptic to the 118 shaded cone synaptic terminals in Figure $1 A$. Foveal cone terminals have $\sim 20$ ribbons (Esfahani et al., 1993; Calkins et al.,

A
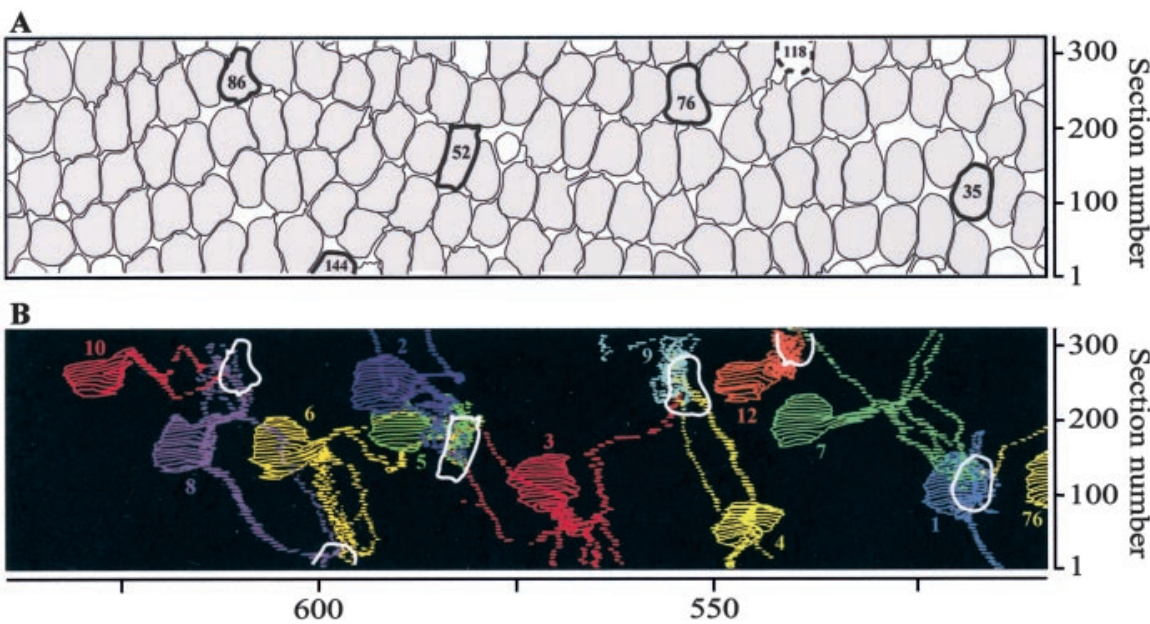

Distance from center of fovea $(\mu \mathrm{m})$

Figure 1. Six S-cone terminals are identified by their connections with $12 \mathrm{~S}$-cone $0 \mathrm{~N}$ bipolar cells. $A$, This map, derived from Tsukamoto et al. (1992), represents a hypothetical horizontal section through the base of the cone terminals, reconstructed from terminals. With five exceptions, each cone terminal contacts a pair of midget bipolar cells, one ON and one OFF. The five exceptional terminals (bold outlines) do not contact ON midget bipolar cells; instead, they contact S-cone ON bipolar cells. The termina bipolar cell; this terminal is identified as that of an S cone on the basis of its contacts with S-cone ON bipolar cells. One 0N midget bipolar cell is two headed and is shared by two L/M cones. $B$, The six S-cone terminals (white outlines) contact $12 \mathrm{~S}$-cone $0 \mathrm{~N}$ bipola cells (colored wire-frame reconstructions). The $S$-cone $0 \mathrm{~N}$ bipolar cell dendrites that are within the series receive synapses

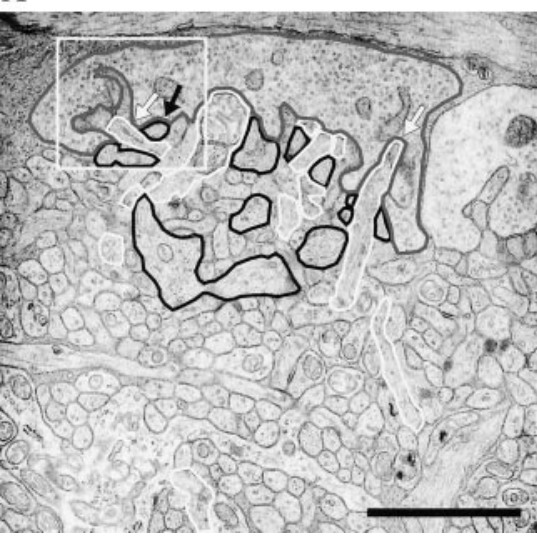

B

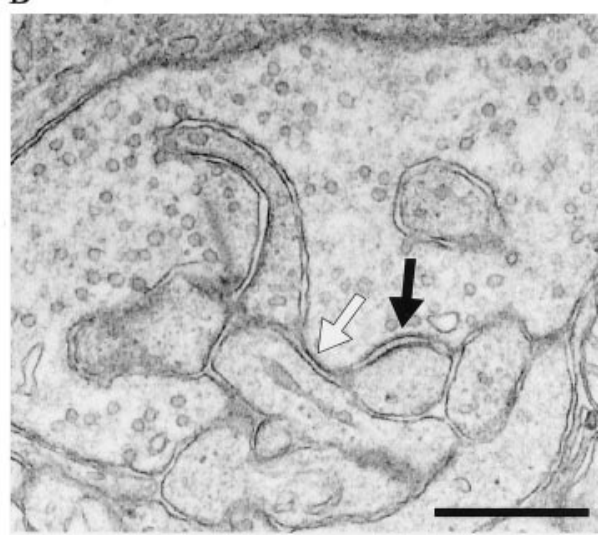

Figure 2. The dendrites of S-cone OFF midget bipolar cells make flat contacts at the triad-associated position. $A$, The synaptic terminal (outlined in gray) of S-cone 35 makes flat contacts with the dendrites of its OFF midget bipolar cell (black outlines) and invaginating contacts with dendrites from several S-cone $0 \mathrm{~N}$ bipolar cells (white outlines). Scale bar, $2.0 \mu \mathrm{m}$. $B$, Enlargement of the boxed area in $A$, showing a symmetrically dense contact (black arrow) with an OFF midget bipolar cell dendrite and an asymmetrically dense contact (white arrow), thicker on the presynaptic side, with an invaginating dendrite from an $\mathrm{S}$-cone $0 \mathrm{~N}$ bipolar cell. Scale bar, $0.5 \mu \mathrm{m}$.

1996), each presynaptic to one or more "triads" of postsynaptic dendrites: two lateral elements, provided by the dendrites of horizontal cells, and one or more central elements, provided by dendrites of invaginating $(\mathrm{ON})$ bipolar cells (Fig. 2 B). Invaginating bipolar cells, whose terminals synapse with ganglion cell dendrites in the inner half of the inner plexiform layer, have ONcenter receptive fields (Famiglietti and Kolb, 1976; Nelson et al., 1978).

We identified all of the ON midget bipolar cells according to the following criteria: (1) their dendrites are postsynaptic to a single cone and (2) provide central elements at ribbon synapses (Kolb, 1970); (3) their synaptic terminals are located in the inner 


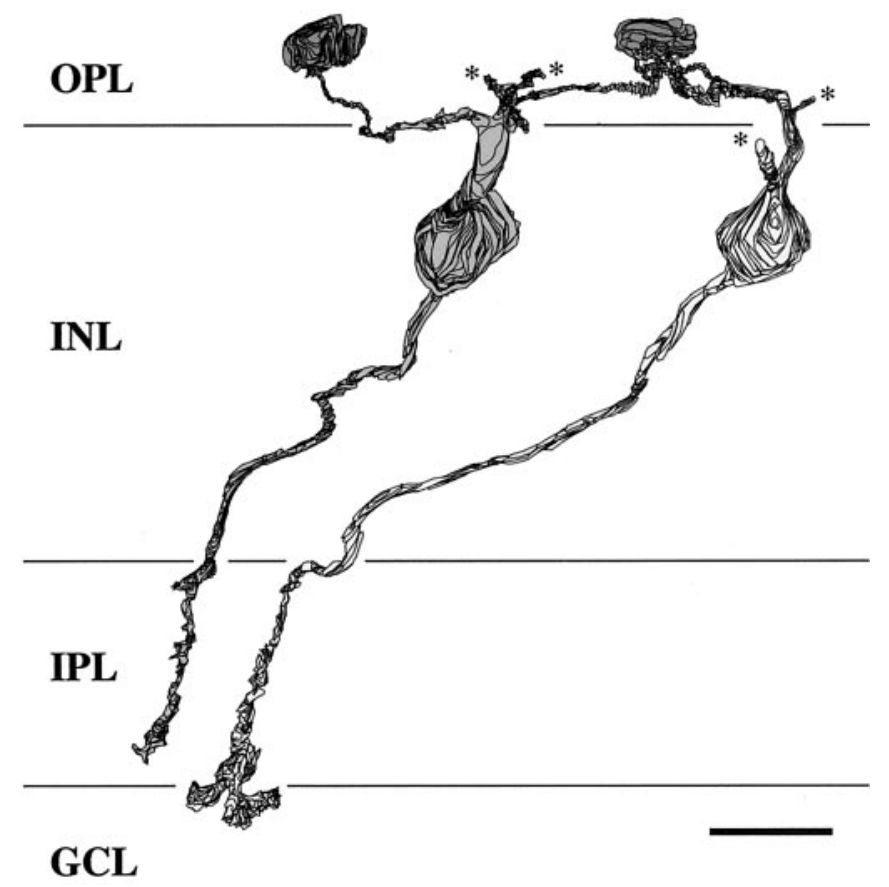

Figure 3. Reconstruction of S-cone $0 \mathrm{~N}$ bipolar cells 3 (red) and 4 (yellow) in Figure $1 B$. The synaptic terminals of these cells are located in the innermost part of the inner plexiform layer, adjacent to the ganglion cell layer. The terminal of the cell on the left is truncated because it extends outside of the series, as do the dendrites marked with an asterisk. INL, Inner nuclear layer; GCL, ganglion cell layer. OPL, outer plexiform layer. Scale bar, $10 \mu \mathrm{m}$.

half of the IPL; and (4) their somas are more densely stained in electron micrographs compared with somas of OFF bipolar cells (Ahmad et al., 2003). One ON midget bipolar cell is "two headed" and is shared by two L or M cones (Polyak, 1941; Hopkins and Boycott, 1992). Of 118 cone terminals, 113 are presynaptic to an ON midget bipolar cell.

Five cones (identified in Fig. $1 A$ ) do not contact $\mathrm{ON}$ midget bipolar cells. The central elements postsynaptic to these five exceptional cones are provided instead by ON bipolar cells whose dendrites branch widely and contact several cones (Fig. $1 B$ ). Unlike the ON and OFF diffuse bipolar cells in our series, which contact all of the cones within a small region, these bipolar cells reach past all intervening cones and contact only the exceptional cones. The synaptic terminals of two of these bipolar cells are contained within the series and are located in the innermost IPL, closer to the ganglion cell layer than the terminals of $\mathrm{ON}$ midget bipolar cells (Fig. 3). These bipolar cells can thus be classified as blue-cone bipolar cells (Mariani, 1984; Kouyama and Marshak, 1992, 1997), and the exceptional cones can be classified as S cones. We rename this bipolar cell type the "S-cone ON bipolar" to distinguish it from other bipolar cells that are postsynaptic to $S$ cones, to indicate that it is depolarized by the onset of shortwavelength light and to emphasize the difference between the spectral sensitivity of S cones and the perceptual quality of blueness. Each S cone contacts several S-cone ON bipolar cells (Fig. 1B) (Herr et al., 2003).

Four of the five S-cone terminals are completely contained within the series and were reconstructed. The number of ribbons in each of the four complete terminals is either 22 or 23, more than that in any other cone terminal in our series (Fig. 4A) (Esfahani et al., 1993). The volumes of the four cone terminals are also among the lowest of all terminals at any given eccentricity (Fig. $4 B$ ). As a consequence, the density of ribbons is highest in
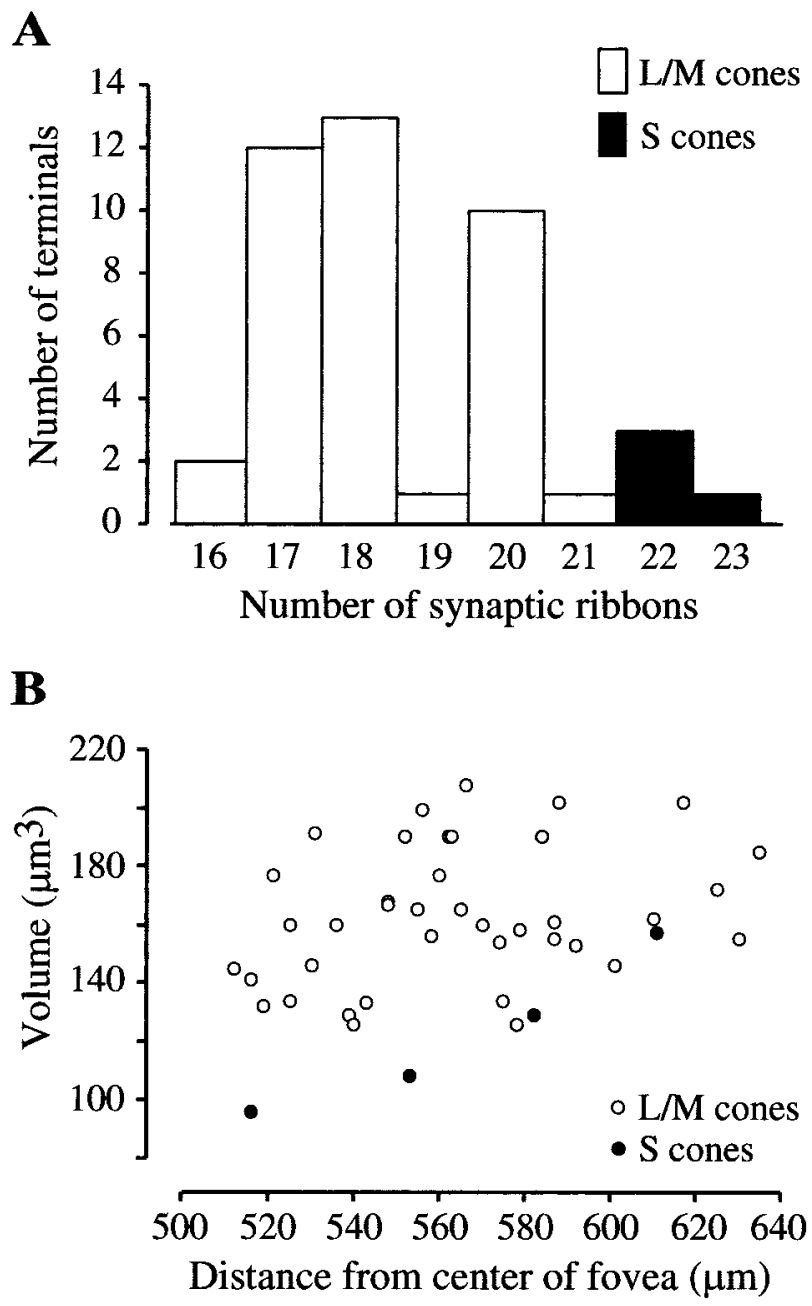

Figure 4. S cones have more ribbons and a smaller cytoplasmic volume than other cones. $A$, Number of ribbons in $39 \mathrm{~L} / \mathrm{M}$ and four $S$ cones $(35,76,52$, and 86$)$. B , Volumes of these 43 cones.

the S-cone terminals, as reported by Ahnelt et al. (1990) and Haverkamp et al. (2001).

A sixth cone terminal (Fig. 1A, 118, dashed outline) contacts two S-cone ON bipolar cells and so was also classified as an $\mathrm{S}$ cone. This cone terminal is only partially contained within the series, so we could not determine conclusively whether or not it contacts an $\mathrm{ON}$ midget bipolar cell. The proportion of $\mathrm{S}$ cones $(6$ of $118, \sim 5 \%$ ) is consistent with other reports of S-cone density at similar eccentricities (Williams et al., 1981; de Monasterio et al., 1985; Curcio et al., 1991; Roorda et al., 2001).

\section{$S$ cones contact OFF midget bipolar cells}

OFF midget bipolar cells postsynaptic to L or M cones: (1) make "flat" contacts with the basal surface of a single cone terminal (Kolb, 1970); (2) have synaptic terminals located in the outer half of the IPL (Famiglietti and Kolb, 1976; Nelson et al., 1978); and (3) appear pale in electron micrographs (Ahmad et al., 2003). This description applies equally well to the subset of OFF midget bipolar cells postsynaptic to $\mathrm{S}$ cones.

The flat synaptic contacts from $S$ cones to OFF midget bipolar cell dendrites are symmetrically thickened (Fig. 2B) (Ahmad et al., 2003) and located near the synaptic ribbon in the triadassociated position, adjacent to contacts with the central elements provided by dendrites of S-cone ON bipolar cells (Fig. 5). The dendritic arbors of two S-cone OFF midget bipolar cells (cells 
35 and 52), reconstructed from highermagnification photomicrographs, receive, respectively, 34 and 35 of these triadassociated contacts from their (S) cone terminals, similar to the number of contacts between some L- and M-cone terminals and their OFF midget bipolar cells (Herr et al., 1995).

We reconstructed two S-cone OFF midget bipolar cells that are completely contained within the sectioned retina. Their synaptic terminals are located in the outer half of the IPL, at the same level as the terminals of OFF midget bipolar cells postsynaptic to non-S cones (Fig. 6). The overall morphology of S-cone OFF midget bipolar cells, as well as finer details of synaptic structure, are indistinguishable from those of OFF midget bipolar cells contacted by $\mathrm{L}$ or $\mathrm{M}$ cones.

All six of the $S$ cones, and all of the other 113 cones in our region, are presynaptic to an OFF midget bipolar cell.

\section{S-cone OFF midget ganglion cells}

Like those of other midget bipolar cells, the synaptic terminals of S-cone midget bipolar cells contain synaptic ribbons (Fig. 7 ), each presynaptic to a "dyad" of postsynaptic elements (Dowling and Boycott, 1966). In almost all cases, one postsynaptic element is provided by a midget ganglion cell and one by an amacrine cell (Calkins et al., 1996). From photomicrographs of the IPL, we reconstructed the synaptic terminals of OFF midget bipolar cells and the dendritic arbors of their midget ganglion cells. The synaptic terminal of each $\mathrm{S}$-cone $\mathrm{OFF}$ midget bipolar cell (Fig. $8 \mathrm{~A}$ ) is enveloped by the dendrites of a single midget ganglion cell (Fig. 8 B).

The synaptic terminals of the two S-cone OFF midget bipolar cells shown in Figure 6 (cells 144 and $35)$ contain, respectively, 30 and 32 synaptic ribbons, similar to the number of ribbons $(32 \pm 3)$ reported for some other OFF midget bipolar cells from the same serial sections (Calkins et al., 1994). The dendrites of the two corresponding OFF midget ganglion cells receive 24 and 29 ribbon synaptic inputs from their bipolar cells, also similar to other midget ganglion cells. The two reconstructed S-cone OFF midget bipolar cells provide the only ribbon inputs to their partner OFF midget ganglion cells, although a small number of dyadic ribbons are presynaptic to dendrites from other, nonmidget, ganglion cells (Calkins et al., 1994).

\section{Discussion}

\section{S-cones and midget bipolar cells}

$\mathrm{S}$ cones are not presynaptic to $\mathrm{ON}$ midget bipolar cells. Instead, as described previously, each S cone is presynaptic to several S-cone ON bipolar cells (Mariani, 1984; Kouyama and Marshak, 1992, 1997), which provide all of the central elements at cone ribbon

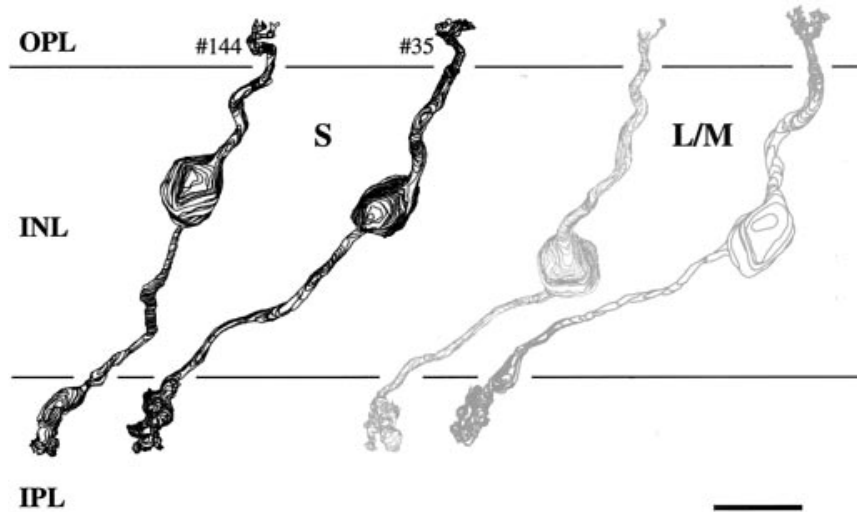

Figure 6. S-Cone OFF midget bipolar cells are indistinguishable from L- and M-cone OFF midget bipolar cells. For comparison, we show reconstructions of two OFF midget bipolar cells contacted by the terminals of S-cones 144 and 35 and two OFF midget bipolar cells contacted by the terminals of $\mathrm{L} / \mathrm{M}$ cones. INL, Inner nuclear layer; $O \mathrm{PL}$, outer plexiform layer. Scale bar, $10 \mu \mathrm{m}$. 
$\mathbf{A}$

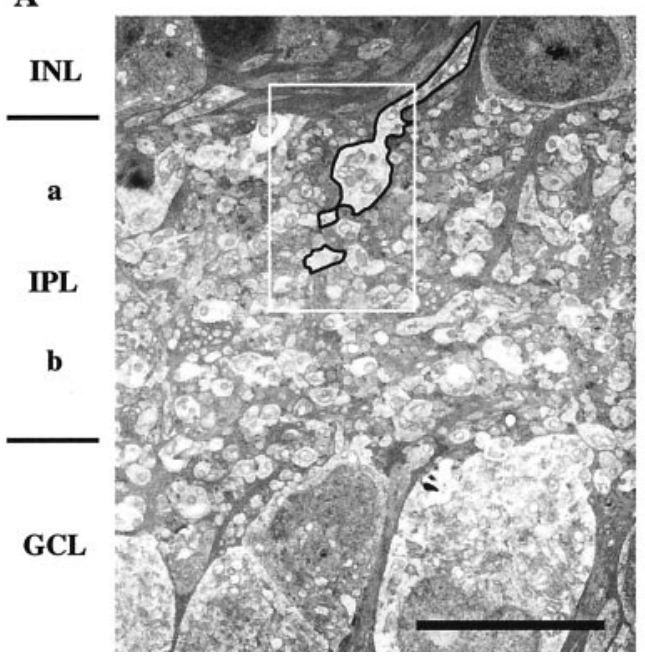

B

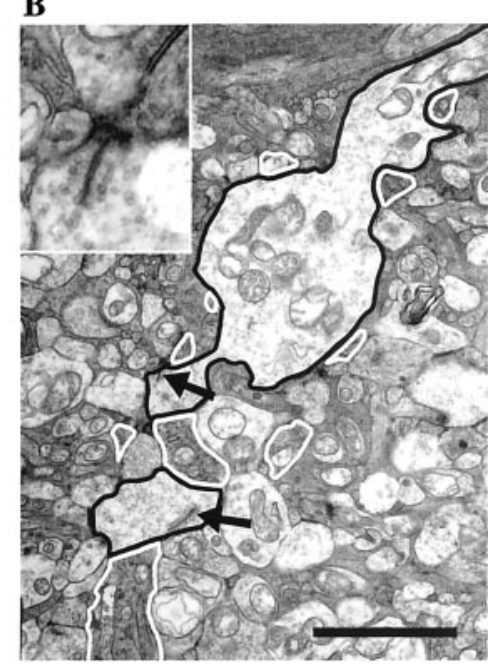

a single $S$ cone, consequently intercepts a small fraction of the light in the blurred short-wavelength spot, whereas L and M cones in the receptive-field ON surround intercept most of the light and might conceal the OFF-center response (de Monasterio et al., 1975). Long-wavelength stimulation would strongly activate the opponent surround, giving the appearance of a large yellow-ON receptive field. $\mathrm{S}$-Cone OFF midget cells might therefore have been misclassified as noncolor opponent (physiological type III) cells (Wiesel and Hubel, 1966; de Monasterio and Gouras, 1975; Dreher et al., 1976; Marrocco, 1976). Supporting this hypothesis, chromatic adaptation by long-wavelength light revealed OFF inputs from $S$ cones to type III cells (Zrenner and Gouras, 1983, their Fig. 3), and selective stimulation of $S$ cones by silent substitution revealed cells with S-cone OFF centers (Derrington et al., 1984; Valberg et al., 1986).

Third, S-cone OFF midget cells might have been misclassified as cells with coex-

synapses (Herr et al., 2003). In contrast, each L and M cone is presynaptic to an ON midget bipolar cell.

$\mathrm{S}$ cones are presynaptic to OFF midget bipolar cells, which in turn contact their own OFF midget ganglion cells. The OFF midget cells of $\mathrm{S}$ cones resemble L- and $\mathrm{M}$-cone OFF midget cells in every feature that we examined, including the following: overall appearance (Fig. 6); symmetric cone synaptic contacts (Fig. $2 B$ ); the number and triad-associated location of those synapses (Figs. $2 B, 5 B$ ); the location of the bipolar cell-ganglion cell synapses in the outer half of the IPL (Figs. 6, 7); the number of ribbons in the synaptic terminal of the bipolar cell; and the exclusive synaptic relationship of the midget ganglion cell with a single midget bipolar cell (Fig. 8).

Kolb et al. (1997) reconstructed S-cone terminals in peripheral human retina but were unable to identify contacts that were clearly presynaptic to an OFF midget bipolar cell. It is possible that peripheral S cones do not contact OFF midget bipolar cells; alternatively, in the work of Kolb et al. (1997), these synapses may have been missed because the bipolar cells were not reconstructed.

\section{S-cone OFF midget circuits are more difficult to record}

Some investigators reported that S-cone OFF-center cells were uncommon in the retina (Malpeli and Schiller, 1978; de Monasterio, 1979; Zrenner and Gouras, 1981; Zrenner, 1983) and LGN (Krüger, 1977; Malpeli and Schiller, 1978). However, De Valois et al. (1966) and Valberg et al. (1986) reported substantial numbers of S-cone OFF cells in the LGN. Because our data suggest that every foveal S cone contacts one OFF midget bipolar cell-midget ganglion cell pair, why did most physiological studies find strikingly fewer S-cone OFF cells than S-cone ON-cells?

First, there are two small bistratified $(\mathrm{ON})$ ganglion cells, but only one OFF midget ganglion cell, for each S cone (Ahmad et al., 2003; Herr et al., 2003). Second, axial chromatic aberration spreads the image of a tiny short-wavelength spot over a large "blur circle" with a diameter similar to the spacing between S cones (Williams et al., 1981; Curcio et al., 1991; Wandell, 1995). The receptive-field center of an S-cone OFF midget cell, driven by tensive receptive-field center and surround (physiological type II) because blurring attributable to chromatic aberration increases the size of an S-cone receptive-field center. In fact, many S-cone OFF ganglion and LGN cells were classified as type II cells (Wiesel and Hubel, 1966, their Fig. 7) (de Monasterio and Gouras, 1975; Reid and Shapley, 2002).

Finally, physiologists generally record outside the fovea, where the proportion of $\mathrm{S}$ cones is lower and midget ganglion cells may receive mixed cone input (Watanabe and Rodieck, 1989; Dacey, 1999, 2000). Consequently, the S-cone input to peripheral midget cells may be overwhelmed by signals from the more numerous $\mathrm{L}$ and $\mathrm{M}$ cones.

\section{Psychophysical evidence for separate $\mathrm{ON}$ and OFF S-cone channels}

In contrast to the mixed physiological results, there is ample psychophysical evidence for the existence of an OFF midget pathway for S-cone signals. Measurements of grating acuity imply that the retina contains a pathway that preserves signals from single foveal S cones (Metha and Lennie, 2001). Shinomori et al. (1999) reported that the threshold for detecting an S-cone stimulus with a rapid-ON sawtooth temporal waveform was elevated more by adaptation to a rapid-ON waveform than to a rapid-OFF waveform and vice versa, suggesting that S-cone signals are carried by two pathways that can be differentially adapted. Using a noisemasking technique to reduce sensitivity separately in the S+ and S- chromatic directions, Sankeralli and Mullen (2001) reported similar results.

Interactions between cone types also suggest separate $\mathrm{ON}$ and OFF pathways. Using transient tritanopia, an elevation of detection thresholds by S cones after the offset of a long-wavelength background, McLellan and Eskew (2000) found that L-cone stimulation in the background affected detection of S-cone increments more than decrements. Color judgments showed a similar asymmetry: the appearance of test patches darker than a surrounding field could be described by a linear combination of S-, $\mathrm{M}$ - and L-cone quantal absorptions, whereas the appearance of test patches lighter than the background required a nonlinear 

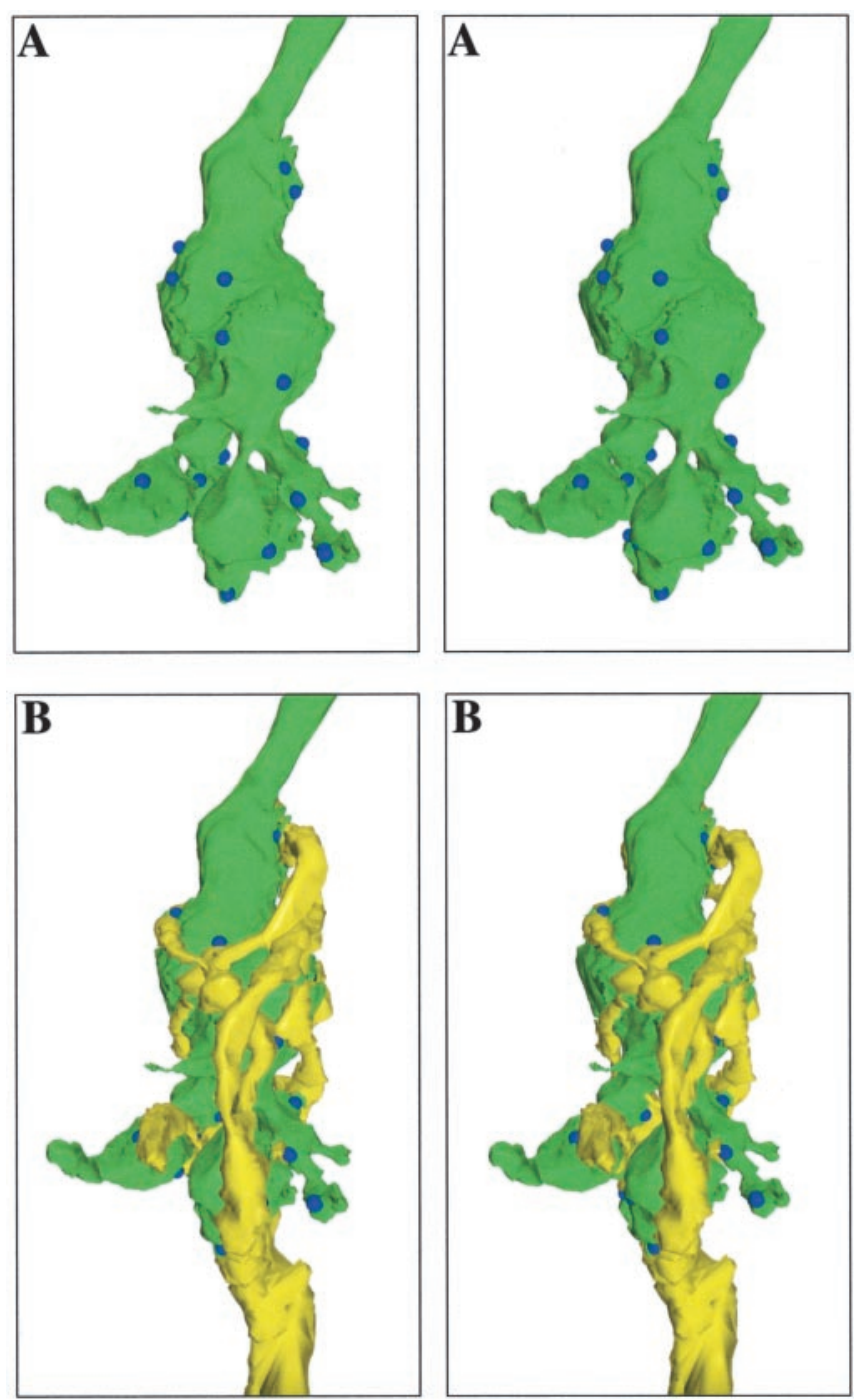

Figure 8. Each S-cone OFF midget bipolar cell terminal connects extensively to one midget ganglion cell. $A$, The synaptic terminal of the OFF midget bipolar cell contacted by S-cone 35 . Blue spheres mark the locations of synaptic ribbons in the bipolar terminal. $B$, The bipolar cell synaptic terminal (green) together with the dendritic arbor (yellow) of its associated midget ganglion cell.

contribution from the S cones (Chichilnisky and Wandell, 1996; Knoblauch and Shevell, 2001).

Finally, inactivating the $\mathrm{ON}$ pathway by exposing the retina to L-2-amino-4-phosphonobutyric acid (L-APB or L-AP-4), which blocks the glutamate receptors of ON but not OFF bipolar cells (Slaughter and Miller, 1981; Schiller et al., 1986), did not change the shape of the spectral sensitivity function, as would be expected if S-cone signals were carried only by ON cells (Smith et al., 1989). Instead, L-APB reduced sensitivity by the same amount at all wavelengths, suggesting the existence of an OFF pathway sensitive to short-wavelength stimuli. Together with other studies demonstrating equal sensitivity to short-wavelength increments and decrements (DeMarco et al., 1994; Schwartz, 1996), the L-APB experiment suggests that the strength of S-cone signals relative to L-and M-cone signals is similar in the ON and OFF pathways.

\section{A role for S-cone OFF midget cells in color perception}

The circuitry of the small bistratified ganglion cell is consistent with the coextensive + S/-L-M physiology required for cells con- tributing to blueness-yellowness (Dacey and Lee, 1994; Calkins et al., 1998; Cottaris and De Valois, 1998; Dacey, 2000; Calkins, 2001). In contrast, no ganglion cell type yet identified has the properties expected for a redness-greenness channel, i.e., coextensive receptive fields driven by $\mathrm{L}$ and $\mathrm{S}$ cones in opposition to $\mathrm{M}$ cones (Hurvich and Jameson, 1957; Wooten and Werner, 1979; Shevell and Humanski, 1988; Rodieck, 1991). It is therefore widely believed that the sensation of redness-greenness is constructed in the visual cortex from signals carried by midget ganglion cells (for an alternative model, see Calkins and Sterling, 1999).

Do S-cone OFF midget bipolar and ganglion cells contribute to these cortically constructed redness-greenness color channels? In particular, do S-cone OFF midget cells contribute to the short-wavelength component of redness? The results of Shinomori et al. (1999) suggest that they do. Their experiments identified separate OFF and ON channels driven by $S$ cones when an adapting sawtooth stimulus left the perception of redness unadapted and available for the detection task, suggesting that both $\mathrm{S}$-cone OFF and ON cells signal redness.

In contrast, S-cone OFF cells do not appear to contribute to blueness: Shinomori et al. (1999) also found that thresholds were determined by the $\mathrm{ON}$ channel alone when an adapting sawtooth left only the perception of blueness available for the detection task.

The hypothesis that S-cone OFF midget cells contribute to redness but not blueness leads to a specific prediction: exposure of the retina to L-APB should block blueness but leave intact an S-cone contribution to redness, causing short-wavelength stimuli to appear red rather than violet.

Sankeralli and Mullen (2001) concluded that yellowness and blueness are carried by separate pathways. Is yellowness signaled by a reduction in the firing rate of S-cone $\mathrm{ON}$ ganglion cells or by an increase in the firing rate of S-cone OFF ganglion cells, or by both? Because L-APB greatly reduces the sensitivity of small bistratified (S-cone ON) ganglion cells to yellow-OFF stimuli (Dacey, 2000), the perception of yellowness should be intact in the presence of L-APB if it is carried by S-cone OFF ganglion cells but will be eliminated or reduced if carried wholly or partly by $\mathrm{S}$-cone ON ganglion cells.

\section{References}

Ahmad KM, Klug K, Herr S, Sterling P, Schein S (2003) Cell density ratios in a foveal patch in macaque retina. Vis Neurosci 20:189-209.

Ahnelt P, Keri C, Kolb H (1990) Identification of pedicles of putative blue sensitive cones in the human retina. J Comp Neurol 293:39-53.

Boycott BB, Dowling JE (1969) Organization of the primate retina: light microscopy. Phil Trans R Soc Lond B Biol Sci 255:109-176.

Calkins DJ (2001) Seeing with S cones. Prog Retin Eye Res 20:255-287.

Calkins DJ, Sterling P (1999) Evidence that circuits for spatial and color vision segregate at the first retinal synapse. Neuron 24:313-321.

Calkins DJ, Schein SJ, Tsukamoto Y, Sterling P (1994) Private lines from M and $\mathrm{L}$ cones in macaque fovea use different numbers of synapses. Nature 371:70-72.

Calkins DJ, Tsukamoto Y, Sterling P (1996) Foveal cones form basal as well as invaginating junctions with diffuse ON bipolar cells. Vision Res 36:3373-3381.

Calkins DJ, Tsukamoto Y, Sterling P (1998) Microcircuitry and mosaic of a blue-yellow ganglion cell in the primate retina. J Neurosci 18:3373-3385.

Chichilnisky EJ, Baylor DA (1999) Receptive-field microstructure of blueyellow ganglion cells in primate retina. Nat Neurosci 2:889-893.

Chichilnisky EJ, Wandell BA (1996) Seeing gray through the ON and OFF pathways. Vis Neurosci 13:591-596.

Cottaris NP, De Valois RL (1998) Temporal dynamics of chromatic tuning in macaque primary visual cortex. Nature 395:845-846.

Curcio CA, Allen KA, Sloan KR, Lerea CL, Hurley JB, Klock IB, Milam AB 
(1991) Distribution and morphology of human cone photoreceptors stained with anti-blue opsin. J Comp Neurol 312:610-624.

Dacey DM (1993) Morphology of a small-field bistratified ganglion cell type in the macaque and human retina. Vis Neurosci 10:1081-1098.

Dacey DM (1999) Primate retina: cell types, circuits and color opponency. Prog Retin Eye Res 18:737-763.

Dacey DM (2000) Parallel pathways for spectral coding in primate retina. Annu Rev Neurosci 23:743-775.

Dacey DM, Lee BB (1994) The "blue-on" opponent pathway in primate retina originates from a distinct bistratified ganglion cell type. Nature 367:731-735.

DeMarco Jr PJ, Smith VC, Pokorny J (1994) Effect of sawtooth polarity on chromatic and luminance detection. Visual Neurosci 11:491-499.

de Monasterio FM (1979) Asymmetry of on- and off-pathways of bluesensitive cones of the retina of macaques. Brain Res 166:39-48.

de Monasterio FM, Gouras P (1975) Functional properties of ganglion cells of the rhesus monkey retina. J Physiol (Lond) 251:167-195.

de Monasterio FM, Gouras P, Tolhurst DJ (1975) Concealed colour opponency in ganglion cells of the rhesus monkey retina. J Physiol (Lond) 251:217-229.

de Monasterio FM, McCrane EP, Newlander JK, Schein SJ (1985) Density profile of blue-sensitive cones along the horizontal meridian of macaque retina. Invest Ophthalmol Vis Sci 26:289-302.

De Valois R, Abramov I, Jacobs GH (1966) Analysis of response patterns of LGN cells. J Opt Soc Am 56:966-977.

Derrington AM, Krausopf J, Lennie P (1984) Chromatic mechanisms in lateral geniculate nucleus of macaque. J Physiol (Lond) 357:241-265.

Dowling JE, Boycott BB (1966) Organization of the primate retina: electron microscopy. Proc R Soc Lond B Biol Sci 166:80-111.

Dreher B, Fukada Y, Rodieck RW (1976) Identification, classification and anatomical segregation of cells with X-like and Y-like properties in the lateral geniculate nucleus of old-world primates. J Physiol (Lond) 258:433-452.

Esfahani P, Schein S, Klug K, Tsukamoto Y, Sterling P (1993) Characterization of L, M. and S cone pedicles in primate fovea. Soc Neurosci Abstr 19:1201.

Famiglietti Jr EV, Kolb H (1976) Structural basis for ON- and OFF-center responses in retinal ganglion cells. Science 194:193-195.

Haverkamp S, Grünert U, Wässle H (2001) The synaptic architecture of AMPA receptors at the cone pedicle of the primate retina. J Neurosci 21:2488-2500.

Herr S, Klug KJ, Sterling P, Schein S (2003) Inner S-cone bipolar cells provide all of the central elements for $\mathrm{S}$ cones in macaque retina. J Comp Neurol 457:185-201.

Herr SS, Tiv N, Klug K, Schein SJ, Sterling P (1995) L and M cones in macaque monkey fovea make different numbers of synaptic contacts with OFF (but not ON) midget bipolar cells. Invest Ophthalmol Vis Sci [Suppl] 36:S8511.

Hopkins JM, Boycott BB (1992) Synaptic contacts of a two-cone flat bipolar cell in a primate retina. Vis Neurosci 8:379-384.

Hurvich L, Jameson D (1957) An opponent-process theory of color vision. Psychol Rev 64:384-404.

Klug K, Tiv N, Tsukamoto Y, Sterling P, Schein SJ (1992) Blue cones contact Off-midget bipolar cells. Soc Neurosci Abstr 18:838.

Klug K, Tsukamoto Y, Sterling P, Schein SJ (1993) Blue cone off-midget ganglion cells in macaque. Invest Ophthalmol Vis Sci [Suppl] 34:S986.

Knoblauch K, Shevell SK (2001) Relating cone signals to color appearance: failure of monotonicity in yellow/blue. Vis Neurosci 18:901-906.

Kolb H (1970) Organization of the outer plexiform layer of the primate retina: electron microscopy of Golgi-impregnated cells. Philos Trans R Soc Lond B Biol Sci 258:261-283.

Kolb H, Boycott BB, Dowling JE (1969) A second type of midget bipolar cell in the primate retina. Philos Trans R Soc Lond B Biol Sci 255:177-184.

Kolb H, Goede P, Roberts S, McDermott R, Gouras P (1997) Uniqueness of the $\mathrm{S}$-cone pedicle in the human retina and consequences for color processing. J Comp Neurol 386:443-460.

Kouyama N, Marshak DW (1992) Bipolar cells specific for blue cones in the macaque retina. J Neurosci 12:1233-1252.

Kouyama N, Marshak DW (1997) The topographical relationship between two neuronal mosaics in the short wavelength-sensitive system of the primate retina. Vis Neurosci 14:159-167.
Krüger J (1977) Stimulus dependent color specificity of monkey lateral geniculate neurons. Exp Brain Res 30:297-311.

Malpeli JG, Schiller PH (1978) Lack of S-cone OFF-center cells in the visual system of the monkey. Brain Res 141:385-389.

Mariani AP (1984) Bipolar cells in monkey retina selective for the cones likely to be blue-sensitive. Nature 308:184-186.

Marrocco RT (1976) Sustained and transient cells in monkey lateral geniculate nucleus: conduction velocities and response properties. J Neurophysiol 39:340-353.

McLellan JS, Eskew Jr RT (2000) ON and OFF S-cone pathways have different long-wave cone inputs. Vision Res 40:2449-2465.

Metha AB, Lennie P (2001) Transmission of spatial information in S-cone pathways. Vis Neurosci 18:961-972.

Meyers D, Skinner S, Sloan K (1992) Surfaces from contours. ACM Trans Graph 11:228-258.

Nelson R, Famiglietti EV, Kolb H (1978) Intracellular staining reveals different levels of stratification for on- and off-center ganglion cells in cat retina. J Neurophysiol 41:472-483.

Polyak SL (1941) The retina, pp 198, 281, 326. Chicago: University of Chicago.

Reid RC, Shapley RM (2002) Space and time maps of cone photoreceptor signals in macaque lateral geniculate nucleus. J Neurosci 22:6158-6175.

Rodieck RW (1991) Which cells code for color? In: From pigments to perception: advances in understanding visual processes (Valberg A, Lee BB eds), pp 83-89. New York: Plenum.

Roorda A, Metha AB, Lennie P, Williams DR (2001) Packing arrangement of the three cone classes in primate retina. Vision Res 41:1291-1306.

Sankeralli MJ, Mullen KT (2001) Bipolar or rectified chromatic detection mechanisms? Vis Neurosci 18:127-135.

Schein SJ (1988) Anatomy of macaque fovea and spatial densities of neurons in foveal representation. J Comp Neurol 266:479-505.

Schiller PH, Sandell JH, Maunsell JH (1986) Functions of the ON and OFF channels of the visual system. Nature 322:824-825.

Schwartz SH (1996) Spectral sensitivity as revealed by isolated step onsets and step offsets. Ophthalmic Physiol Opt 16:58-63.

Shevell SK, Humanski RA (1988) Color perception under chromatic adaptation: red/green equilibria with adapted short-wavelength-sensitive cones. Vision Res 12:1345-1356.

Shinomori K, Spillmann L, Werner JS (1999) S-cone signals to temporal OFF-channels: asymmetrical connections to postreceptoral chromatic mechanisms. Vision Res 39:39-49.

Slaughter MM, Miller RF (1981) 2-amino-4-phosphonobutyric acid: a new pharmacological tool for retina research. Science 211:182-185.

Smith EL, Harwerth RS, Crawford MLJ, Duncan GC (1989) Contribution of the retinal ON channels to scotopic and photopic spectral sensitivity. Vis Neurosci 3:225-239.

Smith RG (1987) Montage: a system for three-dimensional reconstruction by personal computer. J Neurosci Methods 21:55-69.

Tsukamoto Y, Masarachia P, Schein SJ, Sterling P (1992) Gap junctions between the pedicles of macaque foveal cones. Vision Res 32:1809-1815.

Valberg A, Lee BB, Tigwell DA (1986) Neurons with strong inhibitory $\mathrm{S}$-cone inputs in the macaque lateral geniculate nucleus. Vision Res 26:1061-1064.

Wandell BA (1995) Foundations of vision, p 53. Sunderland, MA: Sinauer.

Watanabe M, Rodieck RW (1989) Parasol and midget ganglion cells of the primate retina. J Comp Neurol 289:434-454.

Wiesel TN, Hubel DH (1966) Spatial and chromatic interactions in the lateral geniculate body of the rhesus monkey. J Neurophysiol 9:1115-1156.

Williams DR, MacLeod DI, Hayhoe MM (1981) Punctate sensitivity of the blue-sensitive mechanism. Vision Res 21:1357-1375.

Wooten BR, Werner JS (1979) Short-wave cone input to the red-green opponent channel. Vision Res 19:1053-1054.

Zrenner E (1983) Neurophysiological aspects of color vision in primates. Berlin: Springer.

Zrenner E, Gouras P (1981) Characteristics of the blue sensitive cone mechanism in primate retinal ganglion cells. Vision Res 21:1605-1609.

Zrenner E, Gouras P (1983) Neurophysiological aspects of color vision mechanisms in the primate retina. In: Color vision: physiology and psychophysics (Mollon J, Sharpe L, eds), pp 211-223. New York: Academic. 